\title{
ON AUTOMORPHISM GROUPS OF HOMOGENEOUS COMPLEX MANIFOLDS
}

\author{
SHOSHICHI KOBAYASHI
}

The purpose of this short note is to prove the following:

Theorem. Let $M$ be a homogeneous complex manifold of complex dimension $n$ and $G$ a transitive group of holomorphic transformations of $M$. Let $F$ be the complex Hilbert space of square integrable holomorphic $n$-forms on $M$. Then the natural unitary representation of $G$ in $F$ is irreducible, i.e., no proper closed subspace of $F$ is invariant by $G$.

The reproducing property of the Bergman kernel function [1] plays an essential role in our proof. As we consider not only bounded domains in $C^{n}$, but also general complex manifolds, we replace the kernel function by the kernel form (see [2] for the definition and properties of the kernel form). If $M$ is a domain in $C^{n}$, then $F$ can be identified with the space $F^{*}$ of square integrable holomorphic functions (with respect to the Euclidean measure of $C^{n}$ ). For our purpose, it is, however, desirable to use $F$ even for a domain in $C^{n}$, because every holomorphic transformation of $M$ induces, in a natural way, a unitary transformation of $F$.

We recall that, by a square integrable holomorphic $n$-form $f$, we mean

$$
\left[(-1)^{1 / 2}\right]^{n^{2}} \int_{M} f \wedge \bar{f}<\infty .
$$

The inner product in $F$ is defined by

$$
(f, g)=\left[(-1)^{1 / 2}\right]^{n^{2}} \int_{M} f \wedge \bar{g} .
$$

In general, $F$ may or may not be of infinite dimension, or may even be trivial.

Proof. If $F$ is trivial, that is, $F=\{0\}$, then the theorem is trivially true. We assume therefore that $F$ is nontrivial. Let $\left\{h_{0}, h_{1}, h_{2}, \cdots\right\}$ be a complete orthonormal basis for $F$. Then the kernel form

$$
K(z, \bar{w})=\sum h_{j}(z) \wedge\left\{h_{j}(w)\right\}^{-}
$$

is a holomorphic $2 n$-form on $M \times \bar{M}$, where $\bar{M}$ denotes the complex manifold whose complex structure is conjugate to that of $M$. As in

Received by the editors June $6,1960$. 
the case of the kernel function, $K(z, \bar{w})$ is independent of choice of the basis chosen. By identifying $M$ with the diagonal of $M \times \bar{M}$, we can consider $K(z, \bar{z})$ as a $2 n$-form on $M$. From the nontriviality of $F$ and the homogeneity of $M$, it follows that, for every point $z$ of $M$, there exists an element $f$ of $F$ which does not vanish at $z$. This implies immediately that $K(z, \bar{z})$ is different from zero at every point $z$ of $M$.

Let $F^{\prime}$ be a closed subspace of $F$ invariant by $G$ and $F^{\prime \prime}$ the orthogonal complement of $F^{\prime}$ in $F$. Let $\left\{f_{0}, f_{1}, \cdots\right\}$ (respectively $\left\{g_{0}, g_{1}, \cdots\right\}$ ) be a complete orthonormal basis for $F^{\prime}$ (respectively $\left.F^{\prime \prime}\right)$. Since the kernel form is independent of choice of basis for $F$, we have that

$$
K(z, \bar{w})=\sum f_{j}(z) \wedge\left\{f_{j}(w)\right\}^{-}+\sum g_{k}(z) \wedge\left\{g_{k}(w)\right\}^{-} .
$$

Set

$$
K^{\prime}(z, \bar{w})=\sum f_{j}(z) \wedge\left\{f_{j}(w)\right\}^{-} .
$$

Since $F^{\prime}$ is invariant by $G$, so is $K^{\prime}$. As both $K(z, \bar{z})$ and $K^{\prime}(z, \bar{z})$ are forms of maximum degree on $M$ invariant by $G$ and $G$ is transitive on $M$, we have that

$$
K^{\prime}(z, \bar{z})=c \cdot K(z, \bar{z}) \quad \text { for } z \in M,
$$

where $c$ is a positive constant (provided that $F^{\prime} \neq\{0\}$ ). Since both $K(z, \bar{w})$ and $K^{\prime}(z, \bar{w})$ are holomorphic on $M \times \bar{M}$, we have that

$$
K^{\prime}(z, \bar{w})=c \cdot K(z, \bar{w}) \quad \text { for }(z, \bar{w}) \in M \times \bar{M} .
$$

Take any $f^{\prime \prime}$ from $F^{\prime \prime}$. Then

$$
\int_{\{z\} \times \bar{M}} K^{\prime}(z, \bar{w}) \wedge f^{\prime \prime}(w)=\sum f_{j}(z) \cdot \int_{\bar{M}}\left\{f_{j}(w)\right\}-\wedge f^{\prime \prime}(w)=0
$$

because

$$
\left(f_{j}, f^{\prime \prime}\right)=0 .
$$

On the other hand, we have

$$
\int_{\{z\} \times \bar{M}} K^{\prime}(z, \bar{w}) \wedge f^{\prime \prime}(w)=\int_{[z] \times \bar{M}} c \cdot K(z, \bar{w}) \wedge f^{\prime \prime}(w)=\left[(-1)^{1 / 2}\right] n^{2} c \cdot f^{\prime \prime}(z)
$$

because of the reproducing property of $K$ :

$$
\int_{\{z] \times \bar{M}} K(z, \bar{w}) \wedge f(w)=f(z) \quad \text { for every } f \in F .
$$

Since $c \neq 0, f^{\prime \prime}$ must identically vanish. Hence, $F^{\prime \prime}=\{0\}$. Q.E.D. 


\section{BIBLIOGRAPHY}

1. S. Bergman, Ueber die Kernelfunktion eines Bereiches und ihr Verhalten am Rande, J. Reine Angew. Math. vol. 169 (1933) pp. 1-42, vol. 172 (1935) pp. 89-128.

2. S. Kobayashi, Geometry of bounded domains, Trans. Amer. Math. Soc. vol. 92 (1959) pp. 267-290.

STANFORD UNIVERSITY AND

UNIVERSITY OF British COLUMBIA

\section{SEQUENCES OF HOMEOMORPHISMS ON THE $n$-SPHERE}

M. K. FORT, JR. ${ }^{1}$

1. Introduction. In his recent book of mathematical problems, S. Ulam (see $[1, \mathrm{p} .46]$ ) states the following question as one which he and Borsuk have considered:

Given an arbitrary closed subset $C$ of an $n$-sphere $S, n>0$, does there exist a sequence $H_{1}, H_{2}, H_{3}, \cdots$ of homeomorphisms of $S$ onto itself such that for every $p$ of $S, \lim _{k \rightarrow \infty} H_{k}(p)$ exists and is in $C$, and every point of $C$ is such a limit?

This problem also occurs in the original Scottish Book, along with the remark that Borsuk has solved the problem for the case in which $S$ is two-dimensional.

In this note an affirmative answer is obtained for the above question in the general case. The proof leans heavily upon a result which the author obtained in [2].

2. Admissible polyhedra. Let $\Sigma$ be the set of all closed $n$-cubes which are contained in the euclidean $n$-space $R^{n}$ and whose edges are parallel to the coordinate axes.

If $J \in \Sigma$, a subset $A$ of the boundary of $J$ is an $\alpha$-set of $J$ if $A$ is the union of a collection of $(n-1)$-dimensional faces of $J$ and for some such face $\sigma, \sigma$ is contained in $A$ while the $(n-1)$-dimensional face opposite $\sigma$ is not contained in $A$.

A polyhedron $P$ is admissible if there exists a sequence $P_{1}, \cdots, P_{k}$ of polyhedra such that: $P_{1} \in \Sigma, P_{k}=P$, and for each $i=1, \cdots, k-1$, $P_{i+1}=P_{i} \cup J_{i}$ where $J_{i} \in \Sigma$ and $P_{i} \cap J_{i}$ is an $\alpha$-set of $J_{i}$.

Presented to the Society, August 30, 1960 under the title The limit of a sequence of homeomorphisms of $S^{n}$ onto itself; received by the editors June 22, 1960.

1 The author is an Alfred P. Sloan Research Fellow. 